\title{
Nanopatterned Surface Effect on the Epitaxial growth of InGaN/GaN Multi-quantum Well Light Emitting Diode Structure
}

\author{
Keunjoo Kim* \\ Department of Mechanical Engineering and Research Center of Industrial Technology, Chonbuk National \\ University, Jeonju 561-756, Republic of Korea
}

(Received March 6 2009, Accepted April 23 2009)

\begin{abstract}
The authors fabricated a nanopatterned surface on a GaN thin film deposited on a sapphire substrate and used that as an epitaxial wafer on which to grow an InGaN/GaN multi-quantum well structure with metal-organic chemical vapor deposition. The deposited GaN epitaxial surface has a two-dimensional photonic crystal structure with a hexagonal lattice of $230 \mathrm{~nm}$. The grown structure on the nano-surface shows a Raman shift of the transverse optical phonon mode to $569.5 \mathrm{~cm}^{-1}$, which implies a compressive stress of $0.5 \mathrm{GPa}$. However, the regrown thin film without the nano-surface shows a free standing mode of $567.6 \mathrm{~cm}^{-1}$, implying no stress. The nanohole surface better preserves the strain energy for pseudo-morphic crystal growth than does a flat plane.
\end{abstract}

Keywords: LED, InGaN/GaN, Multi-quantum well, Nanopatterned surface

\section{INTRODUCTION}

A great deal of research has been devoted to the development of InGaN/GaN multi-quantum well (MQW) structures into solid-state lighting sources for use in future illumination systems[1-3]. The spontaneous light emission from these materials is very much dependent on the crystalline quality and, especially, on the threading dislocation originating from the lattice mismatch between the $\mathrm{GaN}$ and the underlying sapphire substrate. A number of attempts have been made to reduce the dislocation effect using such strategies as the insertion of a microscale epitaxial lateral overgrowth (ELOG) layer of $\mathrm{SiO}_{2}$ or a $\mathrm{SiN}_{\mathrm{x}}$ pattern on the GaN thin film [4,5] as well as the use of microscale patterned sapphire substrate (PSS) [6,7]. The reduction of threading dislocations that results from the hetero-epitaxial interface is attributed to stress localization on the buffer layer preventing the residual stress from propagating into the epitaxial layer. All of these different growth methods are intended to localize the interfacial stress.

In the case of the nanoscale patterns, an $\mathrm{SiO}_{2}$ nano-rod array ELOG on a GaN layer enhances the photoluminescence (PL) intensity and reduces the dislocation density[8]. By using a porous alumina template, the resulting nanopatterned surface holes enhance light emission and reduce dislocation density[9]. By decreasing the pattern size to nanoscale, the interfacial stress can be reduced[10]. The inclusions of the ELOG and PSS in the epitaxial growth of the InGaN/GaN MQW structure results in an enhanced PL intensity with a reduced dislocation density [11]. It is interesting to clarify how the misfit stress can be transferred from sapphire substrate to the epitaxial layer via the nanopatterned surface.

In this work, we prepared a nanopatterned $\mathrm{GaN}$ epitaxial wafer grown on a sapphire substrate in order to regrow the InGaN/GaN MQW LED structure. We characterized the

*Author to whom corresponding should be addressed: electronic mail:kimk@chonbuk.ac.kr pseudomorphic epitaxial growth at the nanosurface with micro-Raman spectroscopy and photoluminescence measurements.

\section{EXPERIMENTAL}

Figure 1 shows a schematic diagram of the LED structure including the bottom photonic crystal. On a sapphire wafer covered by a GaN buffer layer, we deposited an n-GaN thin film with metal-organic chemical vapor deposition (MOCVD) in the vertical mode of the reactor, then a periodic hole array with a triangular structure was fabricated on this $\mathrm{GaN}$ substrate using electron beam nanolithography. On top of this GaN substrate prepared with the nanopatterned area, we grew the InGaN/GaN MQW structure of a blue light-emitting diode (LED) using MOCVD. The nanopattern, which is shown in Fig. 2, was fabricated bye-beam lithography and a subsequent ICP process. The lattice constant was $230 \mathrm{~nm}$, while the diameter and the depth of the holes were 120 and $30 \mathrm{~nm}$, respectively.

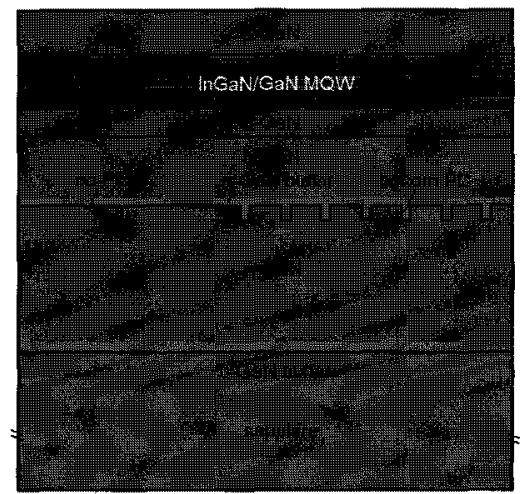

Fig. 1. Schematic diagram of the InGaN/GaN MQW LED structure regrown on nanopatterned surface of $n-G a N$ thin film grown on a sapphire substrate. 

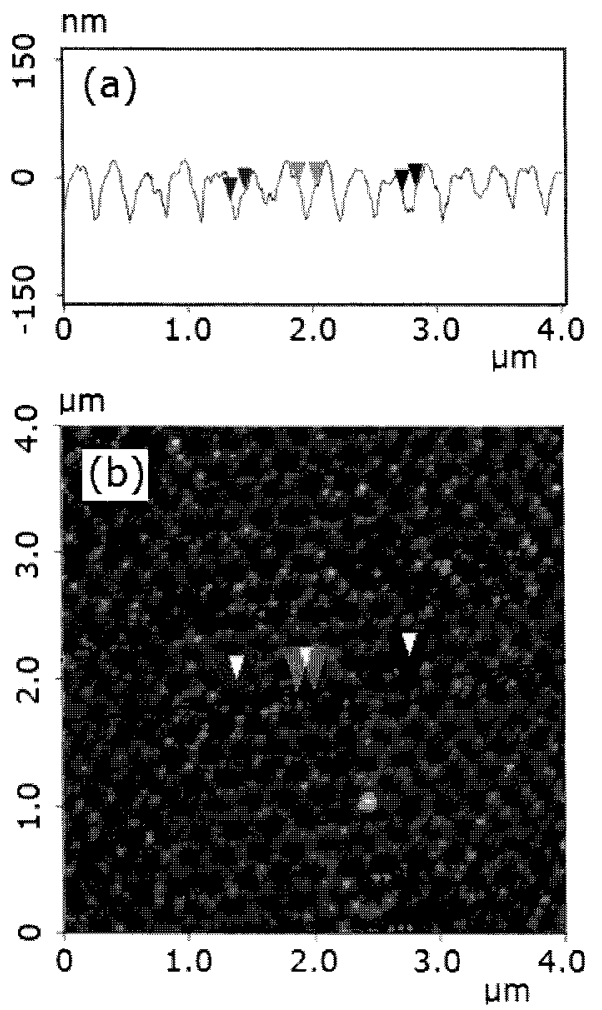

Fig. 2. AFM image of the nanopatterned surface of $n-G a N$ thin film. The nanopattern was processed by e-beam nanolithography with a photonic crystal lattice of $230 \mathrm{~nm}$. The nanoholes have a diameter of $112 \mathrm{~nm}$ and a depth of $30 \mathrm{~nm}$.

The full LED structure was constructed on the nanopatterned epitaxial wafer with multiple epitaxial growths of, in order, a $350-\AA$ GaN buffer layer, a Si-doped n-type GaN layer with a thickness of $1 \mu \mathrm{m}$, a multiquantum well (MQW) active layer, and a Mg-doped p-type layer with a thickness of $0.25 \mu \mathrm{m}$. The five-period InGaN/GaN MQW structure, which served as the blue LED, had a thickness of $20 / 100 \AA$ and In compositions of 0.15 for photoluminescence peaks at $430 \mathrm{~nm}$. A rapid thermal annealing process for p-type activation was carried out at a temperature of $550^{\circ} \mathrm{C}[12]$. The Raman and PL spectra were performed in a backscattering geometry using the $514.5-\mathrm{nm}$ and 457.9-nm lines from an $\mathrm{Ar}^{+}$ion laser and a 325-nm He$\mathrm{Cd}$ gas laser with power at $100 \mathrm{~mW}$ and $30 \mathrm{~mW}$, respectively. The nanostructured surface effect was characterized by a stress analysis derived from the PL and micro-Raman spectra.

\section{RESULTS AND DISCUSSION}

Figure 3 shows the field emission scanning electron microscope (FE-SEM) images of the surface morphology of the GaN epitaxial thin film. Figure 3(a) shows a square surface $300 \mu \mathrm{m}$ across that lies in the nanopatterned area. In the boundary region a high density of threading dislocations can be seen, while there is a relatively low density in the central area. The segregated crystalline grains near the edges were grown outside the nanopatterned area.

Figure $3(\mathrm{~b})$ is a magnified image of a point inside the of nanopatterned area shown in Fig. 3(a). The dislocation density is $4.2 \times 10^{6} \mathrm{~cm}^{-2}$. The growth-pits of dislocation pinholes are hexagonal in shape with the same orientation as the (1122) facets. This phenomenon is similar to what is seen with a lateral overgrowth on PSS[6], as a growing $\mathrm{GaN}$ epilayer on a nanosurface starts to cover the C(0001) plane and nanoholes. The coalesced $\mathrm{GaN}$ buffer layer forms nanovoids inside nanoholes.

Figure 3(c) shows the magnified free standing crystalline grains with dimension smaller than $\sim 100 \mu \mathrm{m}$ that can be seen outside the nanopatterned area in Fig. 3(a). The formation of free standing crystallites indicates that the $\mathrm{n}$ GaN buffer does not provide a 2-dimensional lateral growth condition. GaN homo-epitaxial regrowth on a GaN layer is compressively stressed by a sapphire substrate and a GaN buffer layer without the nanopatterned structure results in a stress relaxation to the substrate-induced misfit strain.

Figure 4 shows the micro-Raman spectra of GaN films regrown on the nanopatterned surface of $n-G a N$ film grown on sapphire substrates. In the nanopatterned area, the Raman intensity is much stronger than in the area without the nanopatterned structure. The spectra show strong $E_{2}(T O)$ high modes under $\mathrm{z}(\mathrm{x},-) \underline{\mathrm{z}}$ scattering geometry. The optical phonon modes of $E_{2}$ (TO) were observed at $567.6 \mathrm{~cm}^{-1}$ from the $n-G a N$ that was regrown on the $n-G a N$ area without a

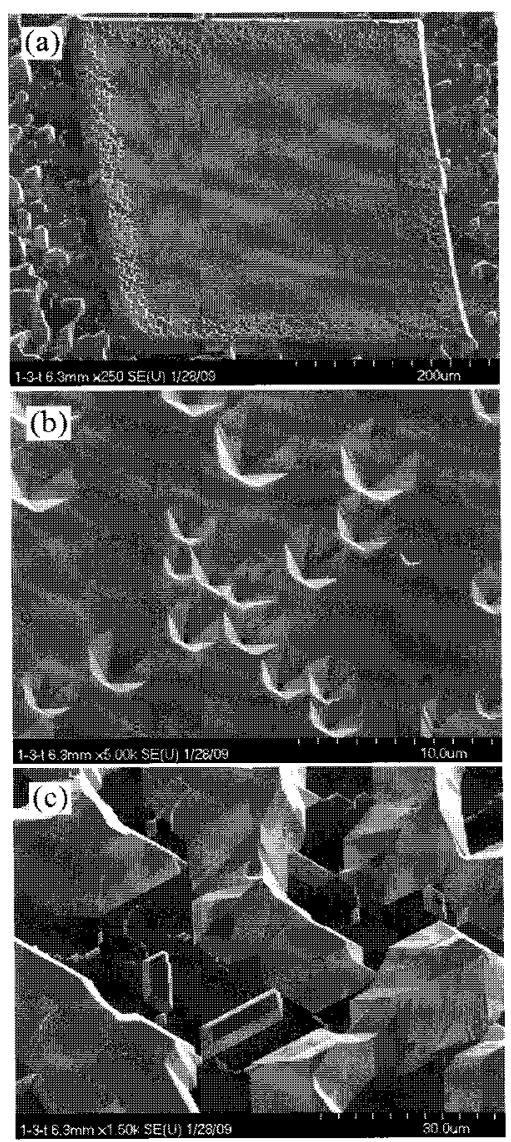

Fig. 3. SEM images of the epitaxially regrown InGaN/GaN MQW LED structure on (a) the nanopatterned surface of $n-G a N$ thin film, (b) an enlarged part of (a), and the regular flat surface without the nanopatterned surface. 


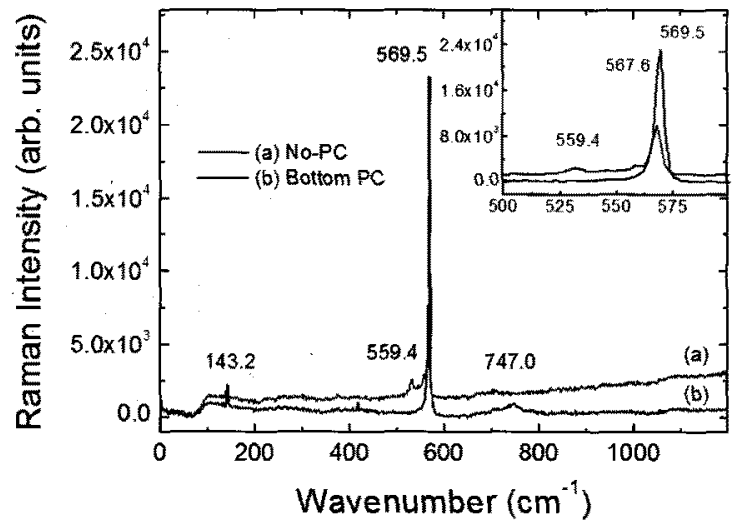

Fig. 4. The Raman spectra of both nanopatterned and regular samples. The regular flat surface provides the home-epitaxial growth for the free standing thin film with the Raman shift of $567.6 \mathrm{~cm}^{-1}$, and the nanopatterned surface shows the value of $569.5 \mathrm{~cm}^{-1}$, indicating that the bottom PC is under the interfacial compressive stress.

nanopatterned surface and at $569.5 \mathrm{~cm}^{-1}$ from the $\mathrm{n}-\mathrm{GaN}$ that was regrown on the nanopatterned $n-G a N$ surface. From a $400-\mu \mathrm{m}$-thick, free-standing, strain-free $\mathrm{GaN}$ layer, the $\mathrm{E}_{2}$ (TO) phonon peak was observed at $567.5 \mathrm{~cm}^{-1}[13,14]$. The nanopatterned surface of $n-G a N$ provides the blue shift for the Raman peak, and the corresponding stress can be analyzed in terms of the lattice constant.

Generally, the type of stress is dependent on the substrate; if the substrate is $\mathrm{Si}(111)$, there is tensile stress, and there is compressive stress when a sapphire substrate is used. The lattice constants of $\mathrm{GaN}, \mathrm{Al}_{2} \mathrm{O}_{3}$, and $\mathrm{Si}$ are 3.189 (5.182), 4.758 (12.991), and $5.43095 \hat{A}$, respectively. For $\mathrm{C}(0001)$ planar growth, the atomic distances of $\mathrm{Ga}-\mathrm{N}, \mathrm{Al}-\mathrm{O}$, and $\mathrm{Si}-\mathrm{Si}$ are $3.189,2.747$ and $3.840 \AA$, respectively, indicating that the sapphire (0001) and $\mathrm{Si}(111)$ substrates produce compressive and tensile stresses on the $\mathrm{GaN}$ epitaxial layer, respectively. Cheong et al.[15] reported that the undoped GaN showed a Raman shift on the $E_{2}(\mathrm{LO})$ phonon at $568.10 \mathrm{~cm}^{-1}$ for the sapphire $(0001)$ substrate, with the phonon peaks of $\mathrm{E}_{\mathrm{g}}$ at 576.56 and $751.10 \mathrm{~cm}^{-1}$. $\mathrm{Mg}$-doped p-type GaN showed phonon peaks of 570.39 and $734.60 \mathrm{~cm}^{-1}$ for $E_{2}(L O)$ and $A_{1}(L O)$ modes, respectively, indicating that p-type doped $\mathrm{GaN}$ is more compressive than the undoped GaN. For a 3- $\mu \mathrm{m}$-diameter, $1.5-\mu \mathrm{m}$-thick micro-pyramidal PSS growth, the Raman shift is about 571 $\mathrm{cm}^{-1}[6]$. However, the introduction of a nanoporous structure with a size range of $20-250 \mathrm{~nm}$ into a GaN layer grown on a sapphire substrate reduces the Raman shift from 568.4 to $567.7 \mathrm{~cm}^{-1}$, indicating a reduction in compressive stress[16]. Therefore, the nanopatterned surface of $n-G a N$ provides a smaller Raman shift than the micro-patterned surface with a reduced biaxial stress.

For the transparent nanopatterned area, the compressive stress from sapphire substrate can be transferred into the home-epitaxial layers of the regrown and undoped GaN, InGaN/GaN MQW, and p-GaN. However, for the reference area without the nanopattern, the compressive stress originating from the sapphire can be relaxed by the GaN buffer layer, and the nucleation points form a free-standing epitaxial growth of crystallites up to the InGaN/GaN MQW

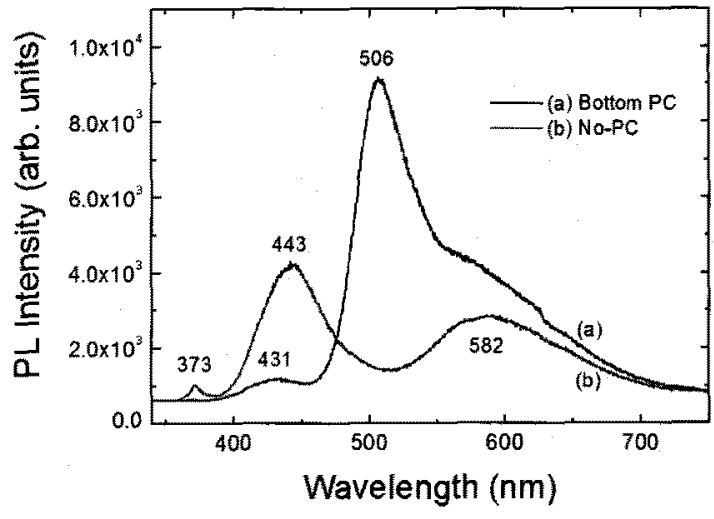

Fig. 5. The PL spectra of nanopatterned and regular samples. The dominant PL peaks at 443 and $506 \mathrm{~nm}$ come from the MQWs, and the broad yellow PL band at $582 \mathrm{~nm}$ originates from the threading dislocation.

layer and the top $\mathrm{p}-\mathrm{GaN}$ layer. The Raman shift rate of $\Delta \omega / \omega$ is related to the in-plane lattice strain $\varepsilon=\Delta a / a$, where a is the lattice constant of $\mathrm{GaN}$. The corresponding strain is $3.35 \times 10^{-3}$, which originates from the lattice mismatch at the $\mathrm{GaN} / \mathrm{sapphire}$ interface. For a Young's modulus of $\mathrm{E}_{<0001\rangle}=150 \mathrm{GPa}[10]$ and Poison's ratio of $v_{<0001>}=0.38[17]$, the Raman shift implies an estimated stress of $0.502 \mathrm{GPa}$.

Figure 5 shows the room-temperature PL spectra of the InGaN/GaN LED samples with and without the inclusion of the GaN nanopattern. The LED sample without the nanopatterned bottom PC surface shows PL peaks at 373 and $443 \mathrm{~nm}$, corresponding to the origins of GaN band-toband edge transition and the quantum well exciton level, respectively. However, the nanopatterned bottom PC LED sample shows PL peaks at 431 and $506 \mathrm{~nm}$. The broad yellow PL band at $582 \mathrm{~nm}$ comes from the influence of the threading dislocation[18].

The reference sample without a bottom PC has an In mole fraction of $x=0.18$ corresponding to a PL peak at 443 $\mathrm{nm}$ for a free-standing InGaN/GaN MQW LED layer. However, the bottom PC sample with the nanopattern has two In fractions of $x=0.16$ and 0.24 for the corresponding PL peaks at 431 and $506 \mathrm{~nm}$, respectively, and it shows a relatively dominant intensity for the spectrum at $506 \mathrm{~nm}$. The PL peak at $431 \mathrm{~nm}$ is similar to the free-standing zone of the reference sample. This indicates that an In mole fraction of $\mathrm{x}=0.18$ in the flat surface of reference sample can be shifted into two phases of In mole fractions: $x=0.16$ and $x=0.24$ in the nanopatterned surface of the bottom PC sample. This phase separation can be correlated with the nanosurface tension and In segregation during the epitaxial growth on the nanopatterned surface of the bottom PC sample. The dominant PL intensity of the $506 \mathrm{~nm}$ shows the increased In mole fraction due to the enhanced solid solution on the nanopatterned surface. It is further desirable to search the electroluminescence spectra on the nanopatterned surface effect.

\section{SUMMARY}

In summary, the influence of the nanopatterned photonic 
crystal effect on photoluminescence was demonstrated in an InGaN/GaN MQW LED structure deposited by MOCVD. The deposited GaN epitaxial surface has been nanopatterned with a hexagonal lattice of $230 \mathrm{~nm}$. The regrown structure shows a Raman shift of transverse optical phonon mode to $569.5 \mathrm{~cm}^{-1}$, which is similar to $\mathrm{n}-\mathrm{GaN}$ thin films grown on a sapphire substrate. However, the regrown thin film without the nanopattern shows a free standing mode of $567.6 \mathrm{~cm}^{-1}$. This result indicates that the nanohole surface plays the role of lattice-fixing points to preserve the strain energy for pseudo-morphic growth. The compressive strain in the nanopatterned $\mathrm{GaN}$ layer can be transferred into the regrown $\mathrm{GaN}$ layer, and the nanopattern provides a better environment for the lateral regrowth of the thin film. To conclude, a nanopatterned surface morphology can provide a good environment for epitaxial growth in spite of the built-in stress-strain effect from the sapphire substrate.

\section{ACKNOWLEDGMENTS}

This work was supported by the Korean Research Foundation Grant(KRF-2007-521-D00188).

\section{REFERENCES}

[1] S. Nakamura and G. Fasol, The Blue Laser Diode: GaN Based Light
Emitters and Lasers, (Springer, Berlin, 1997) p. 203.

[2] G. S. Shin, S. W. Hwang, and K. Kim, Trans. Electr. Electron. Mater. 4, $19(2003)$

[3] K. Kim, Trans. Electr. Electron. Mater. 5174 (2004).

[4] A. Sakai, H. Sunakawa, and A. Usui, Appl. Phys. Lett. 71, 2259 (1997).

[5] T. S. Zheleva, O. H. Nam, M. D. Bremser, and R. F. Davis, Appl. Phys. Lett. 71, 2472 (1997).

[6] D. S. Wuu, W. K. Wang, K. S. Wen, S. C. Huang, S. H. Lin, R. H. Horng, Y. S. Yu, and M. H. Pan, J. Electrochem. Soc. 153, G765 (2006).

[7] T. V. Cuong, H. S. Cheong, H. G. Kim, H. Y. Kim, C.-H. Hong, E. K. Suh, H. K. Cho, and B. H. Kong, Appl. Phys. Lett. 90, 131107 (2007).

[8] C. H. Chiu, H. H. Yen, C. L. Chao, Z. Y. Li, P. Yu, H. C. Kuo, T. C. Lu, S. C. Wang, K. M. Lau, and S. J. Cheng, Appl. Phys. Lett. 93, $081108(2008)$.

[9] J. Park, J.-K. Oh, K.-W. Kwon, Y.-H. Kim, S.-S. Jo, J. K. Lee, and S.-W. Ryu, IEEE Photonics Technol. Lett. 20, 321 (2008).

[10] V. A. Savastenko and A. U. Sheleg, Phys. Status Solidi A, 48, K135 (1978).

[11] D. C. Look and J. R. Sizelove, Phys. Rev. Lett. 82, 1237 (1999).

[12] K. Kim and S. J. Chung, Trans. Electr. Electron. Mater. 2, 24 (2001).

[13] M. Jamil, J. R. Grandusky, V. Jindal, F. Shahedipour-Sandvik, S. Guha, and M. Arif, Appl. Phys. Lett. 87, 082103 (2005).

[14] L. S. Wang, K. Y. Zang, S. Tripathy, and S. J. Chua, Appl. Phys. Lett. 85, 5881 (2004).

[15] T. S. Jeong, C. J. Youn, M. S. Han, J. W. Yang, and K. Y. Lim, Appl. Phys. Lett. 83, 3483 (2003).

[16] H. Hartono, C. B. Soh, S. Y. Chow, S. J. Chua, and E. A. Fitzgerald, Appl. Phys. Lett. 90, 171917 (2007).

[17] T. Detchprohm, K. Hiramatsu, K. Itoh, and I. Akasaki, Jpn. J. Appl Phys, 31, L1454 (1992).

[18] K. Kim and C. B. Park, Thin Solid Films, 330, 139 (1998). 